\title{
Analysis of Seismic Body Waves Excited by the Mount St. Helens Eruption of May 18, 1980
}

\author{
HIROO KANAMORI, JEFFREY W. GIVEN, THORNE LAY
}

Seismological Laboratory, California Institute of Technology

\begin{abstract}
Seismic body waves which were excited by the May 18, 1980, eruption of Mount St. Helens and recorded by the Global Digital Seismographic Network stations are analyzed to determine the nature and the time sequence of the events associated with the eruption. The polarity of teleseismic $P$ waves (period $\sim 20 s$ ) is identical at six stations, which are distributed over a wide azimuthal range. This observation, together with a very small $S$ to $P$ amplitude ratio (at 20 s), suggests that the source is a nearly vertical single force. A simple model shows that for seismic radiation a volcanic eruption can be represented by a single force applied in the direction opposite to the blast direction. The time history of the vertical force suggests two distinct groups of events, about $2 \mathrm{~min}$ apart, each consisting of several subevents with a duration of about $25 \mathrm{~s}$. The magnitude of the force is approximately 2.6 $x 10^{12} \mathrm{~N}$. This vertical force is in contrast with the long-period $(\sim 150 \mathrm{~s})$ southward horizontal single force which has been determined by a previous study and interpreted to be due to the massive landslide. An $M_{s}=5.2$ earthquake initiated the eruption sequence. The direction of the $P$ wave first motion of this event observed at two nearby stations is consistent with the radiation pattern expected for the landslide and suggests that it represents the onset of the landslide. The ground motions observed at station LON $(\Delta-67 \mathrm{~km})$ are dominated by Rayleigh waves (i.e., Lamb pulse) and provide tight constraints on the time sequence of the events.
\end{abstract}

\section{INTRODUCTION}

Kanamori and Given [1982] analyzed long-period ( $200 \mathrm{~s})$ surface waves excited by the eruption of Mount St. Helens on May 18, 1980, and concluded that the source can be adequately represented by a nearly horizontal single force pointed in a direction $55^{\circ} \mathrm{W}$ having a characteristic time constant of about $150 \mathrm{~s}$. They interpreted this single force as due to the massive landslide (total volume - $2.5 \mathrm{~km}^{3}$ ) on the north slope of Mount St. Helens [see Voight et al., 1981]. They also analyzed relatively shortperiod $(-20 s)$ body waves at teleseismic distances and interpreted them in terms of sequences of short-period events during the first several minutes of the eruption. However, because of the very complex and emergent character of the body wave form, detailed analysis could not be made.

While the paper by Kanamori and Given was in press, we were informed by R. Engdahl of the U.S. Geological Survey that a digital recording system was in operation at the World-Wide Standard Seismograph Network (WWSSN) station at Longmire (LON; $\Delta=67 \mathrm{~km}$ and azimuth $=26^{\circ}$ from the summit of Mount St. Helens) and had recorded seismic waves excited by the eruption. On our request, three-component long- and intermediate-period seismograms and a vertical component short-period seismogram from station LON were made available to us by the U.S. Geological Survey. These records provided us with key information regarding the timing of the events and motivated us to perform further analyses of body waves recorded by the Global Digital Seismographic Network (GDSN).

\section{Copyright 1984 by the American Geophysical Union}

Paper number 3B 1597 .

0148-0227/84/003B-1597\$05.00
This paper, which is a follow-up of Kanamori and Given [1982], reports our findings concerning relatively shortperiod events associated with the eruption of Mount St. Helens.

\section{DATA AND ANALYSIS}

\subsection{Far-Field Data}

Figure 1 summarizes the body wave signals recorded by seven GDSN stations. The $P$ waves are recorded at six stations with relatively good azimuthal coverage. The traces are lined up with respect to the Jeffreys-Bullen $P$ arrival times calculated for the origin time of the $M_{s}=5.2\left(m_{b}-4.7\right)$ event (National Earthquake Information Service (NEIS), origin time 1532:11 UT, May 18, $\left.1980 ; 46.214^{\circ} \mathrm{N}, 122.194^{\circ} \mathrm{W}\right)$. The asterisks indicate the Abbreviated Seismological Research Observatory (ASRO) stations; all others are SRO stations. The group delay time of the ASRO instruments is $4 \mathrm{~s}$ longer than that of the SRO instruments at a period of $20 \mathrm{~s}$, which is the dominant period of the observed $P$ waves. Therefore, on Figure 1 the traces of the ASRO stations (KON, ZOB, and MAJ) are moved to the left by $4 \mathrm{~s}$ with respect to those of the SRO stations (GRF, BOC, and GUM). We observe that the waveforms at all stations during the first $3 \mathrm{~min}$ after the $P$ time have the same polarity.

The $S$ waves shown in Figure 1 are the transverse component and are plotted in a manner similar to the $P$ waves. We find that the polarity of the waveforms observed at the stations to the east of the source (i.e., KON, GRF, BOC, and $\mathrm{ZOB}$ ) is reversed with respect to that to the west (i.e., MAJ and TAT). Other important features are that the amplitudes of the $P$ waves are about the same as those of the $S$ waves, and the dominant period of the $S$ waves is about $35 \mathrm{~s}$, which is significantly longer than $25 \mathrm{~s}$, the 


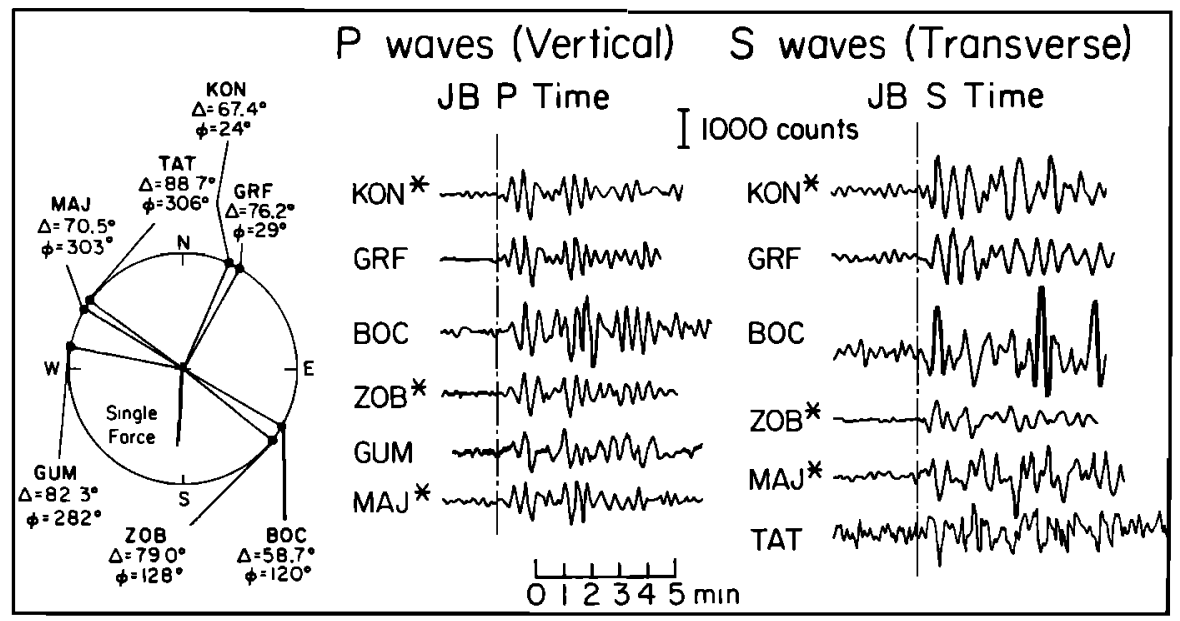

Fig. 1. $P$ waves (vertical component) and $S$ waves (transverse component) excited by the Mount St. Helens eruption and recorded at the GDSN stations. The $P$ and $S$ waves are lined up with respect to the computed JeffreysBullen $P$ and $S$ arrival times, respectively. The stations with and without asterisks indicate ASRO and SRO stations, respectively. The waveforms at ASRO stations are shifted to the left by $4 \mathrm{~s}$ for comparison with those at SRO stations. The vertical scale is for the SRO records. The amplitude of the ASRO records is halved to make it comparable to that of the SRO records. At the period of $25 \mathrm{~s}$, the gain of the SRO instrument is approximately 5000 counts $/ \mu \mathrm{m}$. The distance and azimuth of each station are shown on the left. Note the same polarity of $P$ waves at all the stations.

dominant period of the $P$ waves (see Figure $4 a$ ). The maximum amplitude and the polarity of the $P$ and $S$ waves are plotted in Figure 2 as a function of azimuth. In this plot, the ASRO instrument responses are equalized to the SRO response and a small correction is made for geometrical spreading to normalize the amplitudes to $\Delta-76^{\circ}$, the distance to GRF. The amplitude variation due to the difference in takeoff angle is ignored.

Since the number of stations is very small, we cannot determine the source force system unambiguously from

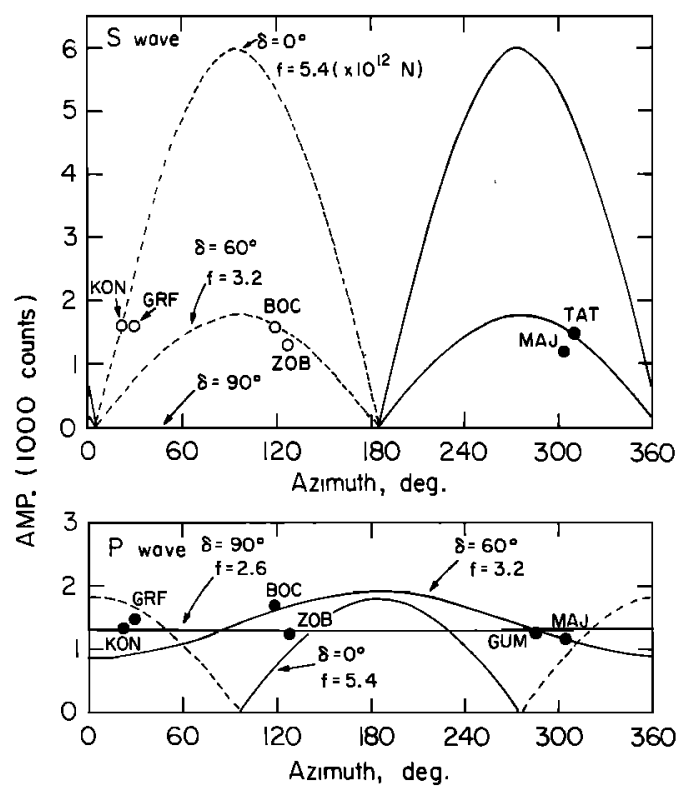

Fig. 2. The variation of the amplitudes as a function of azimuth. The amplitudes are equalized to that of an SRO station at a distance of $76^{\circ}$. Solid and open circles indicate the opposite polarities (see Figure 1). Calculated amplitudes for a single force with dip angles $\delta=0^{\circ}$ and $\delta=60^{\circ}$ are indicated by solid (positive) and dashed (negative) curves; $f$ is the magnitude of the force. this data set. It is difficult, however, to explain the $P$ to $S$ amplitude ratio by using a standard double couple source. Any double couple source would yield $S$ waves with amplitudes several times larger than those of the $P$ waves.

Here we interpret that these short-period waves are due to the explosive sources associated with the eruption. Although the mechanism of seismic radiation by a volcanic eruption is not known in detail, we show in the appendix that a volcanic eruption can be modeled by a combination of a single force representing the thrust of the eruption and an implosive source corresponding to the sudden pressure release in the magma chamber. For a simple model of eruption the amplitude ratio of seismic waves excited by the single force to those excited by the isotropic source is approximately equal to the ratio of the seismic wave velocity to the particle velocity of the fluid (or gas) in the magma chamber. Since this ratio is about 10 , we represent the source by a single force, ignoring the implosive source. Referring to the spherical coordinate system shown in Figure 3, the displacement field at $(r, \theta, \phi)$ in a homogeneous elastic whole space (density, $\rho ; P$ velocity, $\alpha ; S$ velocity, $\beta)$ due to a single force $h(t)(t-$ time) on $x-z$ plane applied at the origin is given by

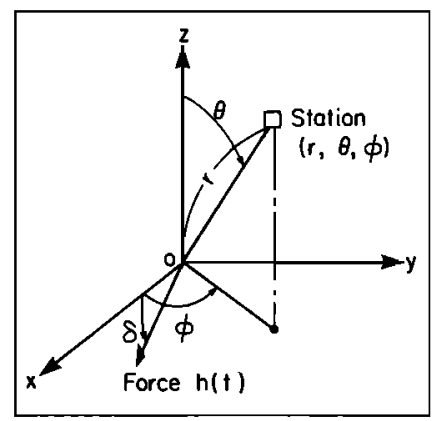

Fig. 3. The coordinate system used in this paper, the geometry of the force, and the location of the station. 

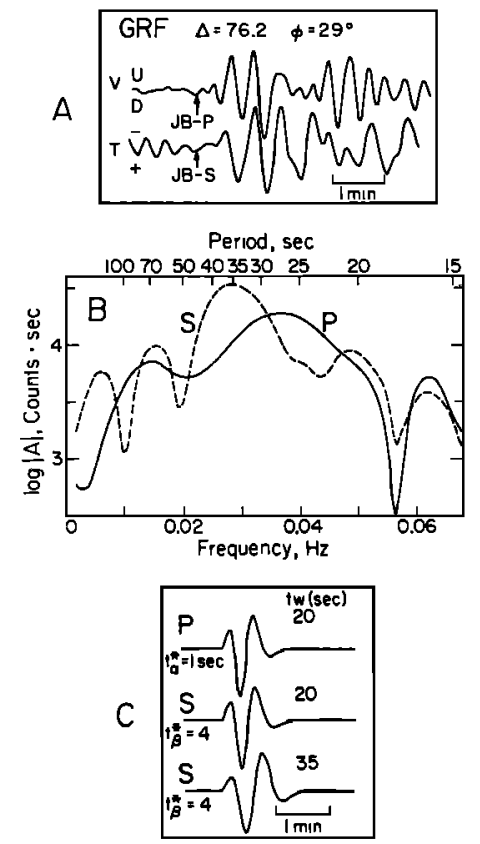

Fig. 4 (a) Comparison of $P$ and $S$ waves observed at station GRF. Note the difference in the period. (b) Frequency spectrum of the $P$ and $S$ waves shown in Figure 4a. (c) Top, synthetic $P$ wave seismograms computed for a single force with a triangular time history: $t_{w}$ is the total width of the triangle, and $t_{\alpha}^{*}-1 \mathrm{~s}$. Middle, synthetic $S$ wave computed for the same source with $t_{\beta}^{*}-4 \mathrm{~s}$. Bottom, synthetic $S$ wave computed for a single force with $t_{w}-35 \mathrm{~s}$. For the computation of synthetic seismograms the dip angle is arbitrarily assumed to be $60^{\circ}$ to the south. Note the almost identical width of $P$ and $S$ waves computed for the same source $\left(t_{w}-20 \mathrm{~s}\right)$. A triangular source with $t_{w}-35 \mathrm{~s}$ can explain the period of the observed $S$ wave.

$$
\begin{aligned}
& {\left[\begin{array}{l}
U_{r} \\
U_{0} \\
U_{\phi}
\end{array}\right]=\frac{1}{4 \pi \rho r \alpha^{2}} h\left(t-\frac{r}{\alpha}\right)\left|\begin{array}{c}
\sin \theta \cos \delta \cos \phi \\
0 \\
0
\end{array}-\cos \theta \sin \theta\right|}
\end{aligned}
$$

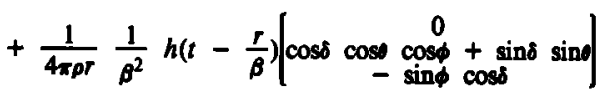

where $\delta$ is the dip angle of the force measured from the horizontal. The angles and $\phi$ are given by $0-\pi-i_{h}$ and $\phi-\phi_{f}-\phi_{s}$, where $i_{h}$ is the takeoff angle of the ray, and $\phi_{f}$ and $\phi_{s}$ are the azimuths of the force and the station, respectively, measured clockwise from the north.

If we assume the same geometry of the force as that determined from the surface waves, that is, $\phi_{f}=185^{\circ}, \delta-0^{\circ}$ (i.e., horizontal force), the radiation pattern becomes two-lobed for both $P$ and $S$ waves, as shown by the curves labeled $\delta-0^{\circ}$ in Figure 2. These curves are calculated with $i_{h}=20^{\circ}$, which corresponds to $\Delta-76^{\circ}$. Since the takeoff angle varies from station to station, we need to apply a small correction to the data to compare them with the computed curve. However, this correction is very small, $\pm 13 \%$ at most, and is not applied here. Figure 2 clearly shows that the horizontal single force cannot explain the constant polarity of the $P$ waves and the amplitude ratio of $P$ to $S$ waves. The magnitude of the force, $f$, assigned to each curve in Figure 2 is determined by using a synthetic seismogram computed for a symmetric triangular source time function having a width of $25 \mathrm{~s}$. The method is described later.

In order to explain the amplitude ratio, the dip angle $\delta$ should be increased as shown in Figure $2 ; \delta=60^{\circ}$ gives an approximately correct $S$ to $P$ ratio as well as a constant $P$ wave polarity. However, this geometry is not satisfactory because, as mentioned earlier, the period of the $S$ waves is much longer than that of the $P$ waves, as shown by Figures $4 a$ and $4 b$. The difference in $Q$ between $P$ and $S$ waves cannot explain this difference in period. Figure $4 c$ compares the waveforms of synthetic $P$ and $S$ waves computed for a symmetric triangular source function having a width $t_{w}$ of $20 \mathrm{~s}$. For this computation, $t_{\alpha}^{*}$ and $t_{\beta}^{*}$ (travel time divided by $Q$ averaged over the path) are assumed to be 1 and $4 \mathrm{sec}$, respectively, the values commonly used in teleseismic body wave studies. The difference in period between the synthetic $P$ and $S$ waves is much smaller than that observed.

Figure $4 c$ also shows a synthetic $S$ wave for a triangular source function with $t_{w}-35 \mathrm{~s}$. A triangular function with $t_{w}=20 \mathrm{~s}$ can explain the dominant period of the $P$ wave, while a width of $35 \mathrm{~s}$ is required to explain the period of the $S$ wave. As shown by Figure $4 b$, the $S$ to $P$ amplitude ratio is even smaller at a period of $25 \mathrm{~s}$ than that indicated by Figure 2; the $S$ waves are essentially at the noise level, and all the stations are considered nodal. This observation, together with the constant amplitude and the uniform polarity of the $P$ waves, suggests that the dip angle of the force is significantly larger than $60^{\circ}$. In the following discussion we use a vertical $\left(\delta-90^{\circ}\right)$ force for simplicity, but the actual dip angle can be in a range from $80^{\circ}$ to $90^{\circ}$.

The source of the $S$ waves remains to be explained. The observed polarity reversal between the groups of the stations to the east and to the west of the source is consistent with the polarity reversal of long-period Love waves [Kanamori and Given, 1982]. This suggests that both the $S$ and Love waves are produced by the same source, a horizontal single force directed in $S 5^{\circ} \mathrm{W}$. In order to investigate this possibility, we band-pass filter the $S H$ waves using a filter described by Kanamori and Stewart [1979] with

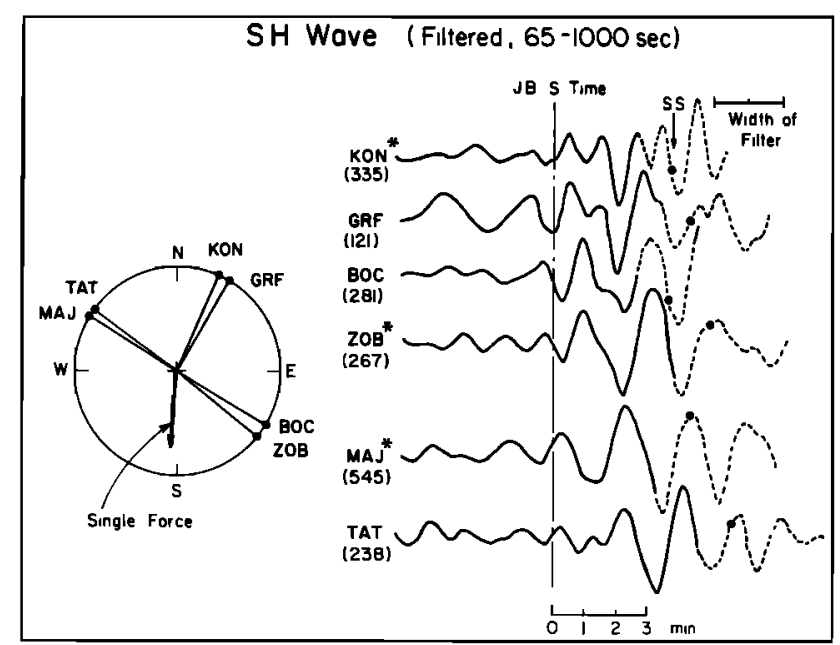

Fig. 5. Filtered $S H$ wave. Note the polarity reversal between stations $\mathrm{ZOB}$ and MAJ. The width of the filter function is indicated in the figure. 


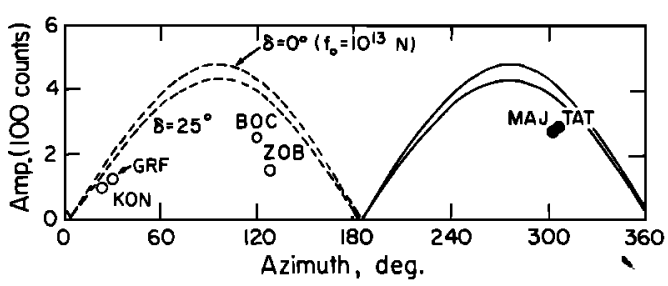

Fig. 6. The azimuthal variation of the amplitude of the filtered $S H$ wave. The amplitude computed for a horizontal single force determined from long-period surface waves is shown by solid and dashed curves.

cutoff periods of 65 and $1000 \mathrm{~s}$. Figure 5 shows the tiltered seismograms, and the maximum amplitudes are plotted in Figure 6 after the difference in geometric spreading factor is corrected. The amplitudes are corrected to a distance of $76^{\circ}$.

In Figure 5, the arrival time of $S S$ is indicated by a dot. Since we are primarily interested in the direct $S$ phase, we indicate by a dashed curve the portion of the filtered records that may be contaminated by the $S S$ phase.

Figures 5 and 6 clearly indicate the polarity reversal between the eastern and the western stations and the nodal character of stations KON and GRF. We compute synthetic seismograms for a single force with the source time history

$$
f(t)=\left\{\begin{array}{cc}
(1 / 2)\left[1-\cos \left(\frac{t}{\tau} \pi\right)\right] & 0 \leqslant t \leqslant 2 \tau \\
0 & t \geqslant 2 \tau
\end{array}\right.
$$

( $\tau$ - 75.0 s) determined by Kanamori and Given [1982]. We assume a homogeneous half space and use the method described by Kanamori and Stewart [1976] with the double couple source replaced by the single force source given by (1). Seismic rays corresponding to $S$ and $s S$ are included, and the same filter as that used for the data is applied. Figure 7 compares the synthetics with the observed (filtered) seismograms. The amplitude of synthetic seismograms computed for $f_{0}-1 \times 10^{13} \mathrm{~N}$, the magnitude of the force determined from long-period surface waves, is plotted as a function of azimuth in Figure 6. Although the synthetic waveforms are similar to the observed, the predicted amplitudes are considerably larger than observed.

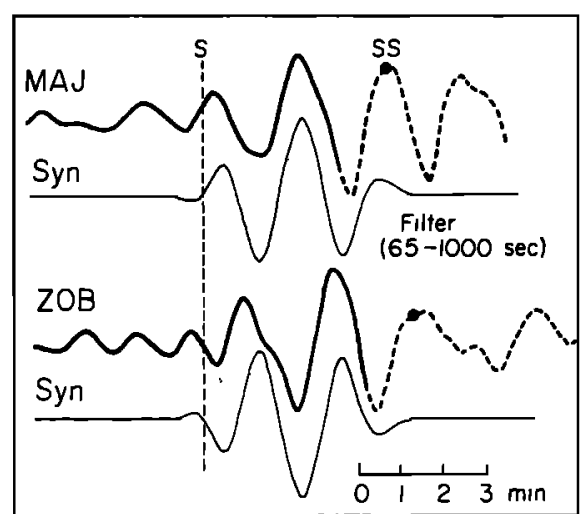

Fig. 7. Comparison of observed and synthetic waveforms of the filtered $S H$ wave. The amplitudes are normalized. The synthetics are computed for a horizontal single force determined from long-period surface waves.
This discrepancy may be due to the difference in the period of the $S H$ waves (about 100 s) shown in Figure 5 and the surface waves (about $200 \mathrm{~s}$ ) used for the determination of the magnitude of the force. It is possible that the shape of the time function (2) is not very accurate and the amplitude of the short-period component is about $70 \%$ smaller than that given by (2), or the geometry of the source at short periods is slightly different from that at long periods. Despite this difference, the approximate agreement in amplitude and polarity indicates that the observed long-period $\mathrm{SH}$ waves are produced by the same source as that for the long-period surface waves. For the same reason, we believe that the $S H$ waves with a period of about $35 \mathrm{~s}$ shown in Figure 1 are due to somewhat irregular components of the horizontal single force.

We next determine the time history of the vertical single force by removing the instrument response from the observed $P$ wave seismograms. Because of the very narrow frequency band of the SRO and the ASRO seismographs, unambiguous determination of the source time history is difficult. Since the waveforms of the observed $P$ waves are essentially identical at all the stations, we take the GRF record and deconvolve it with the instrument response over a relatively narrow period band, 8-100 s. The deconvolved signal is approximated by a series of triangular functions as shown in Figure $8 e$. Then this time function is used to compute the synthetic seismogram for station GRF. A vertical (downward) single force is applied at the surface of a homogeneous half space. We include three rays $P, p P$, and $s P$ from a point source at a finite depth and by making the depth infinitesimally small compute the synthetic for a surface focus. The synthetic seismogram for GRF is shown in Figure $8 b$ with the observed waveform. The same time history is used for the synthetic for station MAJ (Figure $8 d$ ), which is compared with the observed (Figure 8c). The overall agreement between the observed and the synthetic waveforms is satisfactory. We note, however, that because of the narrow-band response of the instrument, the long-period trend cannot be determined, which causes some ambiguity in the polarity of the triangu-

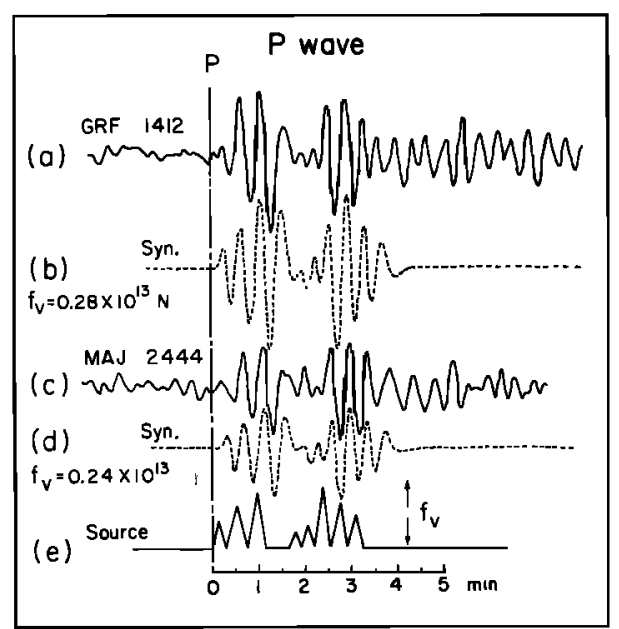

Fig. 8. Comparison of observed and synthetic $P$ waves for stations GRF and MAJ. The synthetics are computed for a vertical single force with the time history shown at the bottom; $f_{v}$ is the peak value of the force. 


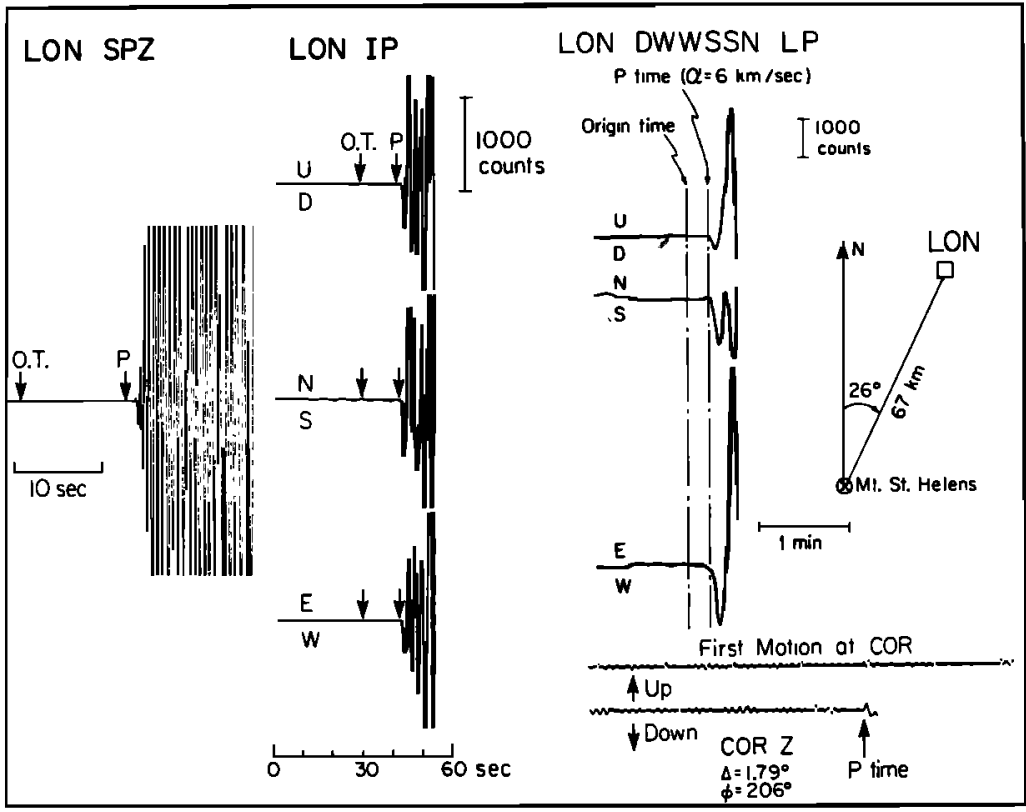

Fig. 9. $P$ wave first motions at stations $L O N$ and COR. For LON the records from long-period (LP), intermediate-period (IP), and short-period (SP) channels are shown, and the origin time of the $M_{s}-5.2$ earthquake and the expected arrival time of the $P$ wave are shown. For COR the vertical component of the WWSSN long-period seismogram is shown, and the expected $P$ time is indicated. Note the upward motion at this time. Geometrical relation of stations LON and COR with respect to Mount St. Helens is also shown.

lar pulses. For example, it is possible to explain the observed waveforms equally well by a series of negative pulses. The seismogram at MAJ has fairly large background noise, and the direction of the first motion is difficult to determine. The first motion of the GRF record is more distinct. If we take the upward motion at about $8 \mathrm{~s}$ after the JB time as the first motion, the polarity of the first triangular pulse should be positive. (Note the delay of the onset of the synthetic seismogram with respect to the onset of the triangular source function. This delay is due to the large group delay time of the SRO and ASRO seismographs.)

Although the details of the source functions cannot be resolved, the triangular source function shown in Figure $8 e$ clearly indicates two distinct groups of events about 2 min apart, each consisting of several subevents. Between the two events, there is a period of quiescence for about $30 \mathrm{~s}$. This character is readily apparent in the original $P$ wave data (Figure 1).

By matching the amplitude of the synthetic and the observed seismograms, we determine the peak value of the single force to be $2.8 \times 10^{12} \mathrm{~N}$ and $2.4 \times 10^{12} \mathrm{~N}$ for GRF and MAJ, respectively (Figure 8 ). The average for all the stations is $2.6 \times 10^{12} \mathrm{~N}$ (see also Figure 2).

A similar analysis is made to determine the magnitude of the force responsible for the short-period $(-35 s)$ component of the $S$ wave. Since the waveform varies from station to station (see Figure 1) after the first cycle, here we attempt to estimate the magnitude of the single force responsible for the first cycle. We find that a symmetric triangular source function having a width of $35 \mathrm{~s}$ can explain the observed period of the $S$ waves. The magnitude of the force is 0.7 to $1.4 \times 10^{12} \mathrm{~N}$ if a horizontal single force is assumed. This value is almost an order of magnitude smaller than that of the long-period component determined from surface waves and long-period $S$ waves. Thus the short-period component represents a very minor perturbation to the time history given by (2).

\subsection{Near-Field Data}

At station Longmire (LON), digital long-period, intermediate-period, and short-period seismograms were obtained. The long-period (DWWSSN LP) records went off-scale about $20 \mathrm{~s}$ after the first arrival, but they clearly indicate a downward first motion (Figure 9). The first motion on the short-period seismogram (DWWSSN SP) is also downward, although it is less clear than the other records (Figure 9). The onset time of the first motion is about $11 \mathrm{~s}$ after the origin time of the $M_{S}=5.2$ earthquake. Since this delay is approximately equal to the travel time of $P$ waves from the summit of Mount St. Helens, we consider that this first motion represents the $P$ arrival of the $M_{s}=5.2$ earthquake, the very beginning of the eruption sequence [Voight et al., 1981].

Figure 9 also shows the first motion at the WWSSN station at Corvallis, which is almost opposite to LON in azimuth (COR, $\Delta-1.79^{\circ}$, azimuth $-206^{\circ}$ ). For the distance of $1.79^{\circ}(199 \mathrm{~km})$ to COR, the $P$ wave travel time is approximately $30 \mathrm{~s}$ and the first motion from the $M_{s}=5.2$ event would arrive at $C O R$ at 1532:41. As Figure 9 shows, the direction of the first motion at COR at this time is up. These data suggest that the $M_{s}-5.2$ earthquake represents the beginning of the sliding motion of the landslide. Since the equivalent force for the landslide is the nearly horizontal southward single force, the first motion would be down at LON and up at COR. However, since no other longperiod data are available, the evidence is not definitive. 


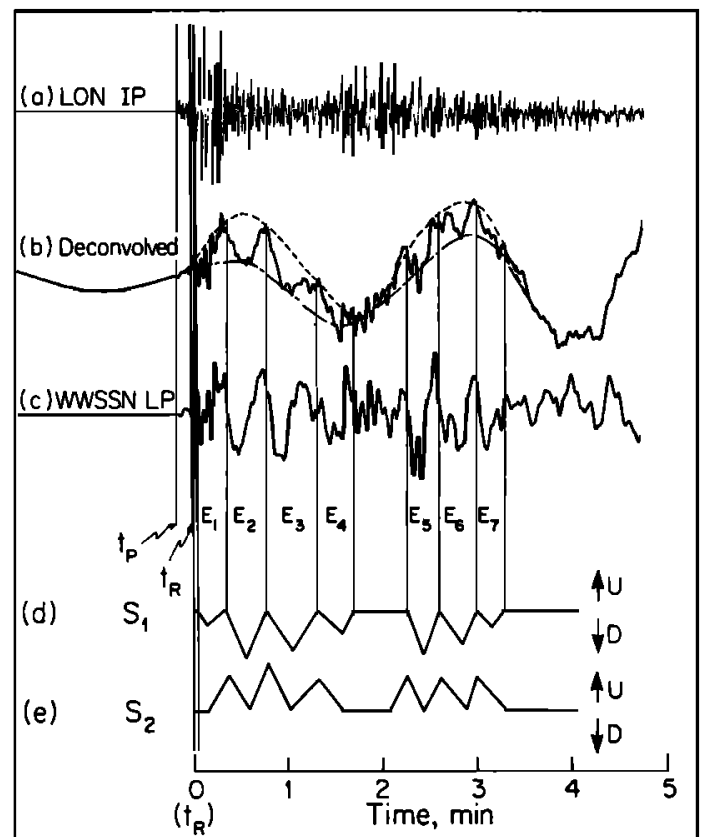

Fig. 10. (a) Intermediate-period seismogram observed at station LON $\left(\Delta-68 \mathrm{~km}, \phi-26^{\circ}\right)$. (b) The displacement time history obtained from Figure $10 a$ by deconvolving the instrument response over a passband from 1.8 to $150 \mathrm{~s}$. (c) WWSSN long-period seismograph response obtained by convolving Figure $10 b$ with the instrument response. (d) Displacement time history obtained from Figure $10 b$ by using the dashed curve as the baseline. (e) Displacement time history obtained from Figure $10 b$ by using the dash-dot curve as the baseline.

Prior to the May 18 eruption, several earthquakes comparable in size to the May 18 event occurred in the same area (April 7, 1980, $m_{b}-4.9$; April 16, 1980, $m_{b}=4.9$; and May 12,1980, $m_{b}-4.8$ ). We examined the WWSSN long-period seismograms of these events recorded at LON and COR. Although the first motion of these events at LON is down, the first motion at COR is not clear enough to determine whether these events have a mechanism similar to that of the event on May 18. Endo et al. [1981] suggest that these events represent rock fracturing due to magmatic pressures.

Since the intermediate-period records of the May 18 event obtained at LON are most complete, we examine them in more detail. First, we deconvolve the instrument response over a passband from 1.8 to $150 \mathrm{~s}$ with the result shown in Figure 10b. The long-period oscillation is caused by the cutoff at the long-period end and is spurious. The relatively short-period oscillations superposed on the longperiod trend represent the real signal. Figure $10 c$ is obtained by convolving the WWSSN long-period response with the deconvolved trace and is equivalent to the standard WWSSN LP seismogram.

We next convolve the deconvolved trace (Figure 10b) with the SRO response to compare it with the far-field waveforms shown in Figure 1 (Figure 11a). A striking similarity between this trace and any one of the far-field records (e.g., GRF, see Figure $11 b$ ) is found during the first $4 \mathrm{~min}$. Figures $11 a$ and $11 b$ are lined up at the $P$ wave arrival time. However, a closer inspection reveals that if we reverse the polarity of Figure $11 a$ and align the start of the trace $9 \mathrm{~s}$ later into the waveform, the waveform simi- larity is even more striking (see Figure 11c). This shift of 9 $s$ is equivalent to matching the $P$ wave arrival time at GRF with the arrival time of a wave with a velocity of $3.1 \mathrm{~km} / \mathrm{s}$ at LON (see Figures $11 b$ and 11c). This result can be explained by using the solution of the classic Lamb's problem [Lamb, 1904]. That is, the LON record represents the Rayleigh wave due to the vertical single force applied at the source. For a step function single force applied vertically downward at the free surface of a homogeneous elastic half space (rigidity $-\mu$ ), the displacement at distance $r$ on the free surface is given approximately by a step function with a delta-function-like singularity preceding it. This step function propagates with the Rayleigh wave velocity, and the amplitude is given by $0.37 / \pi \mu r$ (downward) [e.g., see Richards, 1979, Figure 2c]. When this response is convolved with the instrument response, the delta function pulse has only negligible contribution so that the overall response is given by the step function propagating with the Rayleigh velocity. For a downward vertical force, the main $P$ wave pulse at GRF is upward, while the main Rayleigh wave pulse at LON is downward. Thus the LON record should be reversed in polarity and shifted in time by the amount equal to the difference in the $P$ wave and Rayleigh wave travel times to be matched against the GRF record.

Kanamori and Given [1983] substantiated this interpretation by examining the long-period seismograms recorded at LON for large eruptions on June 13, August 7, and August 8, 1980. They found a clear $90^{\circ}$ phase shift between the vertical and the radial components and confirmed that the observed pulse is a Lamb pulse excited by a vertical downward single force due to the eruption.

Since the waveform of Rayleigh waves at a short distance directly represents the time history of the force at the source, we can use the deconvolved trace at LON to check the result we obtained from the far-field data. As Figure 10 shows, the deconvolved trace indicates a series of pulses, each having a duration of 20-30 s. However, because of the lack of response at long periods, the baseline cannot be determined unambiguously. Here we consider two extreme cases. First, we use the upper envelope shown by a dashed curve in Figure $10 b$ as the baseline. In this case, the displacement is a series of downward pulses as

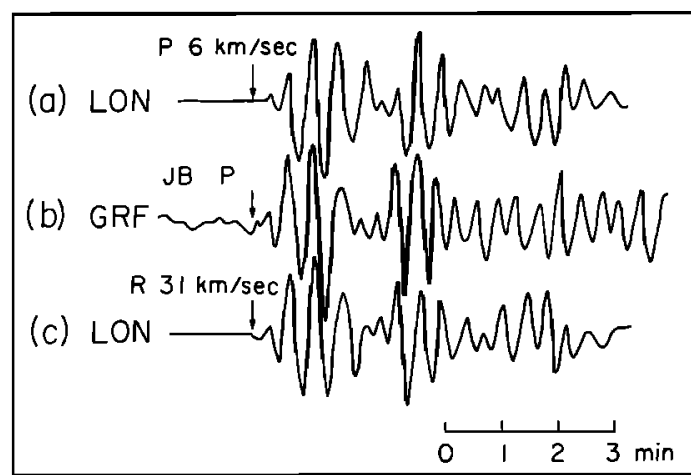

Fig. 11. Comparison of the seismograms recorded at stations (a) LON and (b) GRF. Traces $a$ and $b$ are lined up with respect to the computed $P$ time. (c) Obtained from Figure $11 a$ by reversing the polarity and shifting to the left by $9 \mathrm{~s}$. Note the better waveform match between Figures $11 b$ and $11 c$ than Figures $11 a$ and $11 b$. 


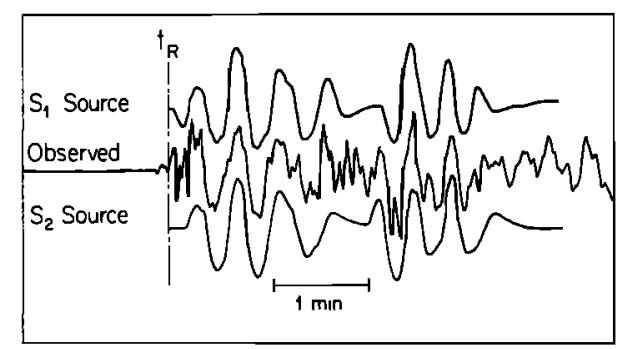

Fig. 12. Comparison of the WWSSN LP response at LON (Figure $10 c)$ and synthetic seismograms computed for the displacement time sequences $S_{1}$ (Figure 10d) and $S_{2}$ (Figure 10e).

shown by $S_{1}$ in Figure $10 d$. The seven subevents represented by the pulses are indicated by $E_{i}(i-1,2, \ldots$ 7). This time series can be interpreted as a sequence of downward vertical forces. Subevent $E_{1}$ starts at, or a few seconds after, the arrival of the Rayleigh wave (indicated by $t_{R}$ ). This event contains a large amount of short-period energy as shown in Figure $10 a$, and $E_{1}$ is followed by two large long-period $(-20 s)$ pulses, the first one beginning at about $22 \mathrm{~s}$ after $t_{R}$. The first sequence of subevents, $E_{1}-E_{2}-E_{3}-E_{4}$ ends at about $1 \mathrm{~min} 40 \mathrm{~s}$ after $t_{R}$ and is followed by a quiet period lasting for about $35 \mathrm{~s}$. The second sequence, which consists of three subevents, $E_{5}-E_{6}-E_{7}$, starts at $2 \mathrm{~min} 15 \mathrm{~s}$ after $t_{R}$. Another notable feature is that during the period between $E_{4}$ and $E_{5}$, when the long-period record indicates quiescence, the amplitude on the short-period record is relatively large.

For the second extreme case, we use the lower envelope indicated by a dot-dash curve in Figure $10 b$ as the baseline.
In this case, the event sequence $S_{2}$ (Figure $10 e$ ) is obtained. Although the overall structure (two events 2 min apart) is similar to that of $S_{1}$, the polarity of the forces is opposite to that of $S_{1}$.

These two event sequences, $S_{1}$ and $S_{2}$, are convolved with the WWSSN instrument response and are compared with the observed trace (Figure 12). Both sequences can explain the overall feature of the observed record satisfactorily. Assuming that the observed signal represents the Rayleigh wave excited by a vertical single force, we can determine the magnitude of the force. Assuming a homogeneous half space with a rigidity $\mu-20 \mathrm{GP}$, we obtain $3.1 \times 10^{12} \mathrm{~N}$ for the peak value of $S_{1}$ and $S_{2}$. This value is about 20\% larger than that obtained from the far-field data but is considered reasonable in view of the very simple half-space model used here. Since the far-field value is determined by the records from many stations, we consider it more reliable and will use it hereafter. However, the LON record probably represents the source time history more accurately than the far-field records.

The actual event sequence is probably somewhere between these two extreme cases. However, the relatively abrupt downward motion at about $2 \mathrm{~s}$ after $t_{R}$ on both the short- and long-period records indicates that the first triangular pulse is probably downward and the sequence given by $S_{1}$ is preferable. The polarity of the later subevents is more difficult to determine. We will use $S_{1}$ for the discussion below, but the uncertainty in the overall polarity should be borne in mind. The event sequence $S_{1}$ is similar to the one determined from teleseismic data shown in Figure 8, although they differ in details.

On the basis of these results, we conclude that two

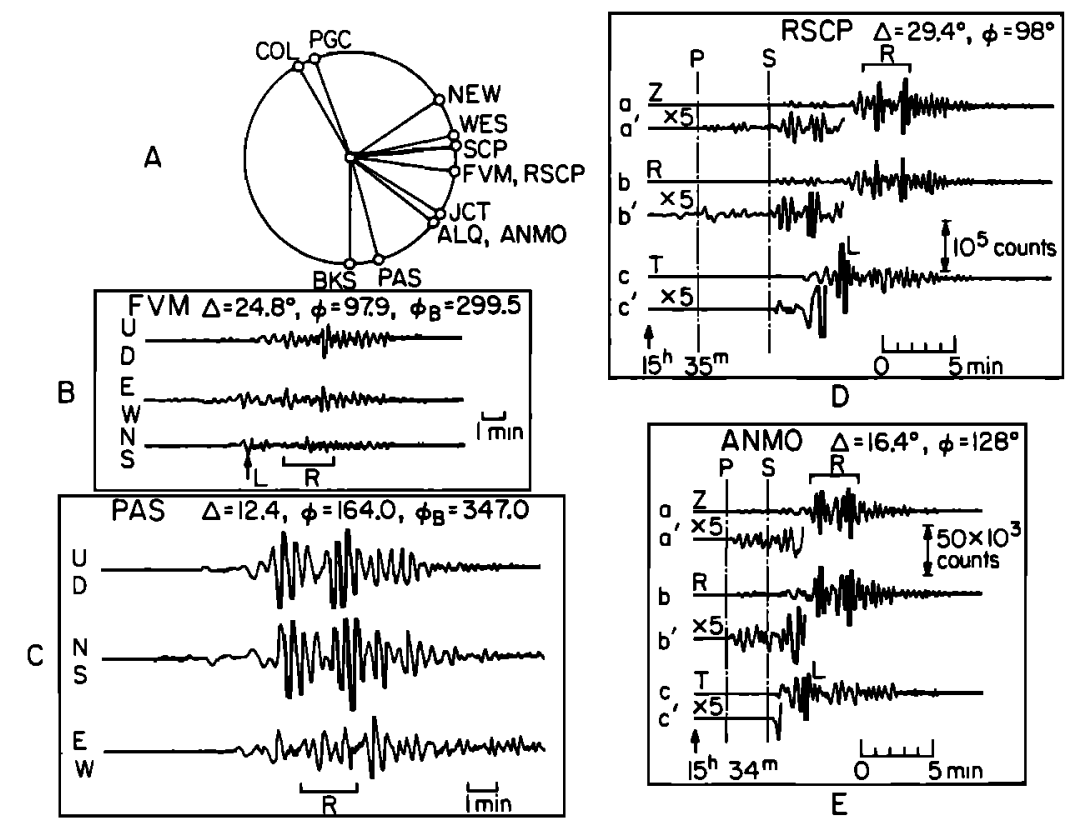

Fig. 13. Seismograms observed at regional stations. (a) Azimuthal distribution of the stations examined. (b) Three-component long-period seismograms at FVM (French Village, Missouri). $L$ and $R$ indicate Love and Rayleigh waves. (c) Three-component long-period seismograms at PAS (Pasadena, California). Note the double arrivals of Rayleigh wave and the absence of Love wave. (d) Vertical (Z), radial (R), and transverse (T) components of the long-period seismograms recorded at RSCP (Cumberland Plateau, Tennessee). Note the distinct double arrivals of the $P$ wave, $S V$ wave, and Rayleigh wave. Also note the small amplitude of the $S H$ wave. (e) Three-component long-period seismograms recorded at ANMO (Albuquerque, New Mexico). 


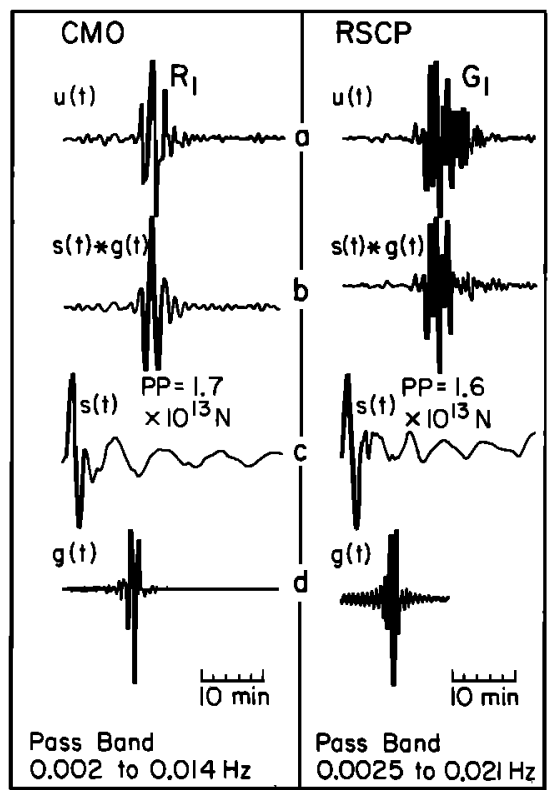

Fig. 14. (left) Determination of the time history of the horizontal force. (a) $u(t)$, long-period Rayleigh wave observed at CMO (College, Alaska); (b) convolution of $s(t)$ and $g(t) ;(c) s(t)$, source time history; $P P$ is the peak-to-peak amplitude; $(d) g(t)$, synthetic seismogram computed for an impulsive single force. (right) Determination of the time history of the horizontal force from Love wave recorded at RSCP (Cumberland Plateau, Tennessee).

major events occurred approximately 2 min apart during the first $4 \mathrm{~min}$. Each event has several subevents. On the LON record the first event begins within a few seconds after $t_{R}$ and contains a relatively large amount of highfrequency energy. Since $t_{R}$ corresponds to the Rayleigh wave travel time from the $M_{s}=5.2$ event, the beginning of the first event is within a few seconds after this earthquake.

\subsection{Regional Data}

The main eruption on May 18 was recorded by many stations in the United States and Canada at distances of less than $40^{\circ}$. The observed phases are mostly short-period $(10-20 \mathrm{~s})$ surface waves. Since quantitative interpretation of these short-period surface waves is difficult because of the complex propagation effects, we try only qualitative analysis of these records. The azimuthal distribution of the stations examined is shown in Figure 13a. The most obvious phase on the record is a Rayleigh wave with distinct double arrivals about $2 \mathrm{~min}$ apart. This Rayleigh wave is seen at all the stations regardless of the azimuth. Examples are shown in Figures $13 b, 13 c, 13 d$, and $13 e$. The double arrivals and the absence of clear azimuthal variation of the amplitudes are consistent with our earlier interpretation that the source of short-period waves is a vertical single force with the time history shown in Figure 10.

Love waves are recorded at stations to the east but not at stations to the south. Unlike the Rayleigh waves, the Love waves do not have the double arrivals (see Figures $13 d$ and $13 e$ ) and are probably excited by the landslide.

The overall nature of the source is best demonstrated by the three-component records at RSCP (Figure 13d), one of the stations of the Regional Seismic Test Network (RSTN). The traces a, b, and $\mathrm{c}$ in Figure $13 d$ show the vertical, radial, and transverse components. The body wave part is blown up 5 times vertically and is shown by traces $a^{\prime}, b^{\prime}$, and $\mathrm{c}^{\prime}$. The $P$ wave, the $S V$ wave (on the vertical and radial components, respectively), and the Rayleigh wave all show the double pulses excited by the vertical force. In contrast, the $S H$ wave is very small, substantiating our conclusion that the short-period source is essentially vertical. As we will show later, the Love wave can be explained by a long-period horizontal single force due to the landslide.

\subsection{Time History of the Long-Period Source}

Kanamori and Given [1982] determined the geometry and time history of the long-period source from longperiod surface waves. They assumed the form of the time function as given by (2) and determined the magnitude of the force and the time constant $\tau$. Here we attempt to determine the time history by directly deconvolving longperiod seismograms recorded at regional as well as teleseismic stations.

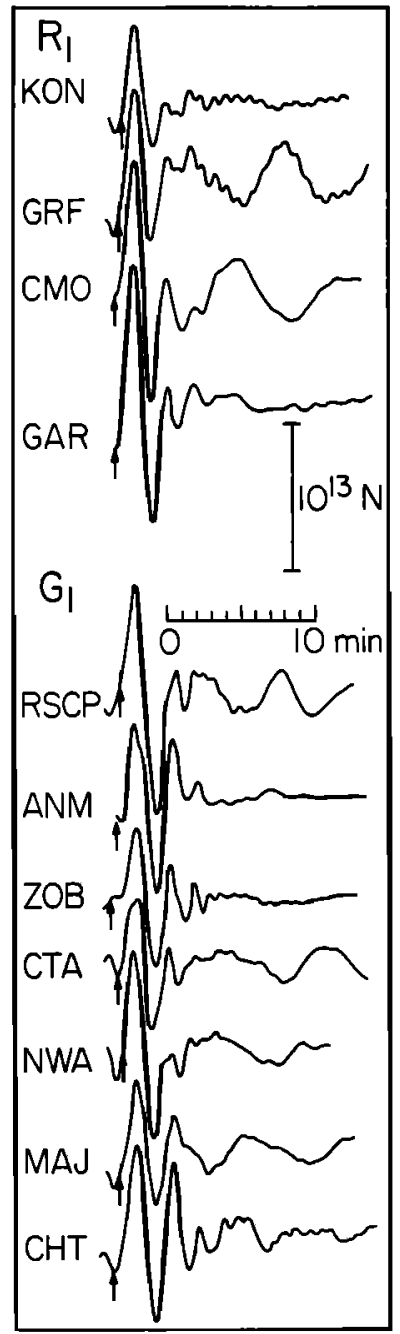

Fig. 15. Source time histories of the horizontal single force determined from Rayleigh waves and Love waves recorded at IDA and GDSN stations. Arrows indicate the origin time. Note the same polarity and approximately the same amplitude and waveform. 


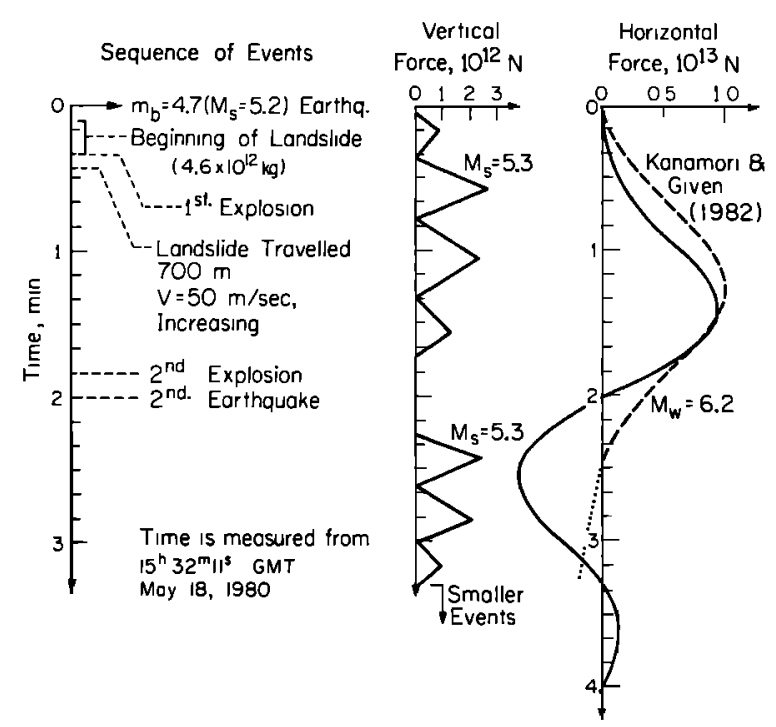

Fig. 16. Sequence of various events associated with the Mount St. Helens eruption and the time history of the long-period horizontal single force and the short-period vertical force. References for the various events are Voight et al. [1981], Glicken et al. [1981], Moore [1981], Moore and Rice [1983], Malone et al. [1981], and Kieffer $[1981 a, b]$.

Let $u(t), g(t)$, and $s(t)$ be the observed seismogram, the synthetic seismogram computed for an impulsive force, and the time history of the force, respectively. Then $u(t)=g(t) *_{s}(t)$ where the asterisk is the convolution operator. In the frequency domain, $\hat{u}(\omega)-\hat{g}(\omega) \cdot \hat{s}(\omega)$, where $\hat{u}(\omega)$, etc., are the Fourier transforms of $u(t), g(t)$, and $s(t)$ respectively. We determine $\hat{s}(\omega)$ by dividing $\hat{u}(\omega)$ by $\hat{g}(\omega)$ over a limited frequency band and inverse transform it to obtain the time history $s(t)$. Using the method described by Kanamori [1970], we compute $g(t)$ for the force geometry determined by Kanamori and Given [1982]. Figure 14 (left) shows an example. The Rayleigh wave train $u(t)$ recorded by an International Deployment of Accelerographs (IDA) station at CMO $\left(\Delta=23.4^{\circ}\right.$, azimuth $-332^{\circ}$ ) is shown by Figure $14 a$ (left). The impulse response $g(t)$ is shown by Figure $14 d$. Figure $14 c$ is the source time function $s(t)$ obtained by deconvolution of $u(t)$ over a frequency band from 0.002 to $0.014 \mathrm{~Hz}$. Figure $14 b$ is the convolution $g(t) * s(t)$, which reproduces the observed seismogram $u(t)$ reasonably well. Because of the limited band width of the instrument, the lateral heterogeneity of the earth's structure, and the noise in the record, the deconvolved source time history $s(t)$ is subject to some errors. The very long-period (about $500 \mathrm{~s}$ ) oscillation seen on Figure $14 c$ is the noise caused by the cutoff at the low-frequency end of the spectrum. However, the main pulse during the first $5 \mathrm{~min}$ of $s(t)$ is a robust feature that does not change for changes in the passband over a reasonable range. Since the distance to CMO is only $23.4^{\circ}$, the effect of the propagation path on the waveform is small. We therefore consider that $s(t)$ shown in Figure 14 (left) is a good representation of the actual time history.

We make a similar analysis on the Love wave recorded at $\operatorname{RSCP}\left(\Delta-29.4^{\circ}\right.$, azimuth $-98^{\circ}$, Figure 14 (right)). The source time function $s(t)$ obtained from this record is very similar to that obtained from the Rayleigh wave recorded at CMO.
We analyze nine more phases, from IDA and GDSN stations, and the results are shown in Figure 15. We use a passband from 0.002 to $0.014 \mathrm{~Hz}$ for IDA stations (CMO and GAR), and 0.0025 to $0.021 \mathrm{~Hz}$ for GDSN stations. Since the distances to some of the stations are very long, the source functions obtained from those stations are less accurate than those obtained from nearby stations such as CMO and RSCP. Nevertheless, all the source time functions have the same polarity and approximately the same amplitude and waveform. Since the long-period response of IDA stations is much better than that of GDSN stations, we consider the result obtained from the CMO record most reliable. As shown in Figure 16, this time history is very similar, for the first $2 \mathrm{~min}$, to that determined by Kanamori and Given [1982]. An overshoot occurs at 2 min after the origin time. Since this overshoot is seen for most stations shown in Figure 15, it is probably a real feature of the source time history.

\section{DISCUSSION}

The time histories of the vertical force determined from the far-field and near-field data are shown in Figure 16 together with that of the long-period horizontal single force determined from the CMO record. Some results from various on-site observations are also included.

Although the time history of the forces determined here can be used as a relatively objective constraint for various models of the eruption sequence, its interpretation is not unique. Here, we attempt to provide one possible interpretation on the basis of the various seismological observations reported in this paper. Since the interpretation of the long-period signals in terms of the landslide is discussed in detail by Kanamori and Given [1982], here we focus on the short-period events.

According to the descriptions and reconstructions of the eruption sequence by various investigators [Voight et al., 1981; Moore and Albee, 1981; Malone et al., 1981], the sequence during the first several minutes can be generally described as follows.

The eruption on May 18, 1980, began as a large seismic event. Within 7 to no more than $20 \mathrm{~s}$ after this event, the north slope began to slide northward. As the slide developed and progressed, steam and magmatic explosions began due to pressure release behind the slide scarp, and the slide became engulfed in the resulting blast cloud. A second large earthquake occurred about 2 min after the first.

Although we could not determine the mechanism of the initial $M_{s}=5.2$ event unambiguously, the first-motion data at LON and COR suggest that this earthquake may represent the beginning of the sliding motion of the landslide. The first event $E_{1}$ (see also Figure 10), which contains a relatively large amount of high-frequency energy, may represent the initial stage - of the eruption before the vent had been fully uncapped. Events $E_{2}, E_{3}$, and $E_{4}$ can be interpreted as due to the explosions that were triggered by removal of the overburden pressure. The relatively quiet period after $E_{4}$ may represent the end of the initial series of explosions; the significance of the increased amplitude of the short-period 
waves shown in Figure $10 a$ during this quiet period is not clear. Events $E_{5}, E_{6}$, and $E_{7}$ appear to represent the second sequence of explosions described by Moore and Rice [1983]. Moore and Rice report, on the basis of measurements from infrared sensors aboard two U.S. Air Force satellites and of ground photographic and eyewitness records, that a second explosion occurred a few kilometers north of the first, about $1.5 \mathrm{~min}$ later. Moore and Rice [1983] conclude that this second explosion was the largest of the eruptions and caused most of the damage and tree blow down.

The magnitude of the force, about $2.6 \times 10^{12} \mathrm{~N}$, is comparable to the value, $3.3 \times 10^{12} \mathrm{~N}$, estimated by Kieffer $[1981 a, b]$ using a steady flow model for a multiphase (vapor-solid-liquid) mixture expanding from the vent. However, the orientation of the force determined from the seismic data is nearly vertical, while the model used by Kieffer [1981a] is for a lateral blast. The short-period $S$ wave data (Figure 1) indicate some horizontal component $\left(-1 \times 10^{12} \mathrm{~N}\right)$, but it can be due to either the irregular motion of the landslide or the horizontal component of the force due to the blast. As discussed by Kanamori and Given [1982], the horizontal force is dominated by a very long period component.

It should also be noted that a part of the seismic excitation could be due to some processes that occurred within the magma chamber and may not be directly related to the surface eruption.

\section{CONCLUSION}

The radiation pattern of $P$ and $S$ waves (period 20-30 s) excited by the May 18, 1980, Mount St. Helens eruption and their amplitude ratio can be explained by a nearly vertical single force at the source with a magnitude of about $2.6 \times 10^{12} \mathrm{~N}$. The time history of this vertical force suggests two distinct groups of events each consisting of several subevents with a duration of about $25 \mathrm{~s}$. The two groups are separated in time by approximately $2 \mathrm{~min}$. This vertical force is in contrast with the long-period (duration $>150 \mathrm{~s}$ ) horizontal single force with a magnitude of $10^{13}$ Kanamori and Given [1982]. We interpret that this vertical force is due to the explosions. Although this vertical force contributes to the radiation of long-period Rayleigh waves, its effect is negligible, since the long-period component of the vertical force is only about $10 \%$ of the horizontal force.

Although the direction of the first motion of the $M_{s}$ 5.2 earthquake at 1532:11 UT is ambiguous at teleseismic stations, it is clearly recorded at two stations at short distances, LON and COR. The first motion is up at COR ( $\Delta$ $\left.-199 \mathrm{~km}, \phi-206^{\circ}\right)$ and down at LON $(\Delta=68 \mathrm{~km}$, $\phi-26^{\circ}$ ), which is opposite in azimuth to COR. This pattern of the first motion is consistent with that expected for the southward horizontal single force and suggests, though not definitely, that the $M_{s}=5.2$ earthquake represents the onset of the sliding motion of the landslide. If this interpretation is correct, gravitational instability caused by the preeruption bulging of the north slope near the summit of Mount St. Helens resulted in spontaneous outbreak of the massive landslide.

The ground motion observed at LON can be interpreted as Rayleigh waves excited by the vertical single force associated with the eruption and is considered a Lamb pulse. The waveform of this Lamb pulse provides constraints on the time sequence of the events during the first $3 \mathrm{~min}$ after the beginning of the eruption and on the magnitude of the force.

\section{APPENDIX: A FORCE SYSTEM EQUIVALENT to a Volcanic ERUPTION}

A force double couple is widely used as a force system equivalent to earthquake faulting. However, there is no well-established force system to represent a volcanic eruption. In what follows, we show that a single force is adequate to represent a simple model of volcanic eruption.

Consider a pressurized cylinder (pressure $P$, height $l$, and radius a) embedded in an elastic half space (Figure $\mathrm{A} 1 a$ ). The cylinder is initially capped by the lid AB, which is removed at time $t=0$ to cause an "eruption." Before the eruption, the fluid (or gas) inside the cylinder exerts the force $F_{T}-\pi a^{2} P$ on the lid $\mathrm{AB}$, which, in turn, pulls the half space upward. When the lid AB is suddenly removed, the force vanishes instantaneously, while the pressure inside the cylinder decreases to zero gradually, with a time constant $\tau$. This time constant is at least comparable to $l / v$, where $v$ is the particle velocity of the fluid inside the cylinder. Since the forces acting on the sidewall, $F_{S}$, and the bottom, $F_{B}$, of the cylinder are proportional to the pressure, they also decrease gradually to zero. Figure $\mathrm{A} 1 b$ schematically shows the time histories of these forces.

We then decompose $F_{T}$ into two components, $F_{T}$ and $F_{T_{2}}$, in such a way that $F_{T_{2}}$ has the same time history as $F_{B}$ (Figure $\mathrm{Al} c$ ). The force $F_{T}$ is a vertical downward force. The forces $F_{T_{2}}, F_{B}$, and $F_{S}$ form an implosive source. If $l$ $-2 a$, we can approximate the implosive source by an isotropic implosive source consisting of three orthogonal dipoles with a moment $M-3 \pi a^{3} P$ [Love, 1934, p. 187].

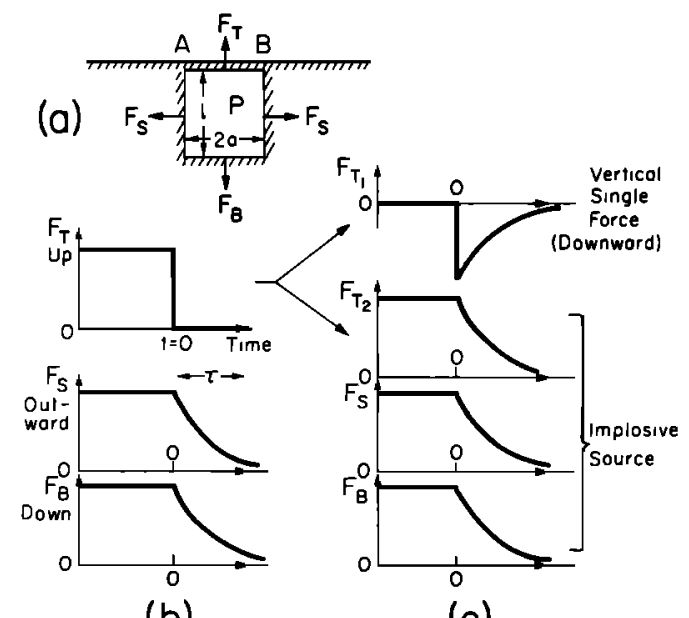

(b)

(c)

Fig. A1. A force system equivalent to a volcanic eruption. ( $\alpha$ ) A pressure release model for a volcanic eruption. (b) Forces acting on the top, side, and bottom walls. $(c)$ Decomposition of the force to a vertical single force and an implosive force. 
The maximum amplitudes of the seismic waves at farfield (distance $r$ ) due to the isotropic source and the single vertical force are

$$
\left|U_{I}\right|-\left|\frac{M}{4 \pi \rho c^{3} r}\right|-\left|\frac{3 \pi a^{3} P}{4 \pi \rho c^{3} r} \frac{1}{\tau}\right|
$$

and

$$
\left|U_{F}\right|-\left|\frac{F}{4 \pi \rho c^{2} r}\right|-\left|\frac{\pi a^{2} P}{4 \pi \rho c^{2} r}\right|
$$

respectively, where $P$ and $c$ are the density and the seismic wave velocity of the elastic medium. The ratio of $U_{I}$ to $U_{F}$ is then

$$
U_{I} / U_{F}-3 a / c \tau-(3 / 2)(v / c)
$$

Since $\mathrm{v} \sim 100 \mathrm{~m} / \mathrm{s}$ and $\mathrm{c} \sim 3 \mathrm{~km} / \mathrm{s}$, we can ignore the effect of the isotropic source.

In the near-field,

$$
\left|U_{I}\right|-\left|\frac{F a}{4 \pi c^{2} r^{2}}\right|
$$

and

$$
\left|U_{F}\right|-\left|\frac{F}{4 \pi c^{2} r}\right|
$$

so that

$$
\left|U_{I} / U_{F}\right|-|(a / r)|
$$

Thus as long as $(a / r)<<1$, the effect of the isotropic source can be ignored.

Acknowledgments. We thank Robert Engdahl and John Hoffman of the U.S. Geological Survey for making available to us the digital seismograms used in this study. We thank Holly Eissler for helping us retrieve the records of the RSTN network. This research was partially supported by National Aeronautics and Space Administration grant NSG-7610 and National Science Foundation grant EAR811-6023. Contribution 3854, Division of Geological and Planetary Sciences, California Institute of Technology, Pasadena, California 91125.

\section{REFERENCES}

Endo, E. T., S. D. Malone, L. L. Noson, and C. S. Weaver, Locations, magnitudes, and statistics of the March 20-May 18 earthquake sequence, in The 1980 Eruptions of Mount St. Helens, edited by P. W. Lipman and D. R. Mullineaux, U.S. Geol. Surv. Prof. Pap., 1250, 93-107, 1981.

Glicken, H., B. Voight, and R. J. Janda, Rockslide-debris avalanche of May 18, 1980, Mount St. Helens volcano, paper presented at the International Association of Volcanology and Chemistry of the Earth's Interior Symposium, Tokyo, Aug. 28-Sept. 9, 1981.

Kanamori, H., Synthesis of long-period surface waves and its application to earthquake source studies, Kurile Islands earth- quake of October 13, 1963, J. Geophys. Res., 75, 5011-5027, 1970.

Kanamori, H., and J. W. Given, Analysis of long-period seismic waves excited by the May 18, 1980, eruption of Mount St. Helens - A terrestrial monopole?, J. Geophys. Res., 87, $5422-5432,1982$.

Kanamori, H., and J. W. Given, Lamb pulse observed in nature, Geophys. Res. Lett., 10, 373-376, 1983.

Kanamori, H., and G. S. Stewart, Mode of strain release along the Gibbs fracture zone, Mid-Atlantic Ridge, Phys. Earth Planet. Inter., 11, 312-332, 1976.

Kanamori, H., and G. S. Stewart, A slow earthquake, Phys. Earth Planet. Inter., 18, 167-175, 1979.

Kieffer, S. W., Blast dynamics at Mount St. Helens on 18 May 1980, Nature, 291, 568-570, $1981 a$.

Kieffer, S. W., The lateral blast of May 18 at Mount St. Helens, paper presented at the International Association of Volcanology and Chemistry of the Earth's Interior Symposium, Tokyo, Aug. 28-Sept. 9, $1981 b$.

Lamb, H., On the propagation of tremors over the surface of an elastic solid, Philos. Trans. $R$. Soc. London Ser. A, 203, 1-42, 1904.

Love, A. E. H., A Treatise on the Mathematical Theory of Elasticity, 4th ed., 643 pp., Cambridge University Press, New York, 1934.

Malone, S. D., E. Endo, C. S. Weaver, and J. W. Ramey, Seismic monitoring for eruption prediction, in The 1980 Eruptions of Mount St. Helens, edited by P. W. Lipman and D. R. Mullineaux, U.S. Geol. Surv. Prof. Pap., 1250, 803-813, 1981.

Moore, J. G., The pyroclastic surge of May 18, 1980, Mt. St. Helens, Washington, paper presented at the International Association of Volcanology and Chemistry of the Earth's Interior Symposium, Tokyo, Aug. 28-Sept. 9, 1981.

Moore, J. G., and W. C. Albee, Topographic and structural changes, March-July 1980--Photogrammetric data, in The 1980 Eruption of Mount St. Helens, edited by P. W. Lipman and D. R. Mullineaux, U.S. Geol. Surv. Prof. Pap., 1250, 123-141, 1981.

Moore, J. G., and C. J. Rice, Chronology and character of the May 18, 1980, explosive eruptions of Mount St. Helens, in: Explosive Volcanism: Inception, Evolution, and Hazards, Geophysics Study Committee, Nat. Acad. Press, Washington, D.C., Chapter 10, in press, 1983.

Richards, P. G., Elementary solutions to Lamb's problem for a point source and their relevance to three-dimensional studies of spontaneous crack propagation, Bull. Seismol. Soc. Am., 69, 947-956, 1979.

Voight, B., H. Glicken, R. J. Janda, and P. M. Douglass, Catastrophic rockslide-avalanche of May 18, in The 1980 Eruptions of Mount St. Helens, edited by P. W. Lipman and D. R. Mullineaux, U.S. Geol. Surv. Prof. Pap., 1250, 347-378, 1981.

J. W. Given, H. Kanamori, and T. Lay, Seismological Laboratory, California Institute of Technology, Pasadena, CA 91125.

(Received January 3, 1983;

revised August 19, 1983;

accepted September 22, 1983.) 